\title{
Repression of interferon regulatory factor 3 by the Epstein-Barr virus immediate-early protein Rta is mediated through E2F1 in HeLa cells
}

\author{
LIANG-HUA ZHU ${ }^{1}$, SHAN GAO ${ }^{1}$, RUI JIN ${ }^{1}$, LI-LI ZHUANG ${ }^{1}$, LI JIANG ${ }^{1}$, \\ LING-ZHI QIU ${ }^{1}$, HUA-GUO XU ${ }^{2}$ and GUO-PING ZHOU ${ }^{1}$ \\ Departments of ${ }^{1}$ Pediatrics and ${ }^{2}$ Clinical Laboratory, The First Affiliated Hospital, \\ Nanjing Medical University, Nanjing, Jiangsu 210029, P.R. China
}

Received July 7, 2013; Accepted February 10, 2014

DOI: $10.3892 / \mathrm{mmr} .2014 .1957$

\begin{abstract}
Interferon regulatory factor 3 (IRF-3), an essential transcriptional regulator of the interferon (IFN) genes, is important in the host defense against viral and microbial infection. Epstein-Barr virus (EBV) immediate-early protein replication and transcription activator (Rta) and the transcription factor E2F1 are two important inhibitive factors, which repress IRF-3 expression. Numerous studies have identified that Rta can directly bind to the Rta-response element in promoters of its target genes and regulate their expression. In the present study, we demonstrated that Rta represses the expression of IRF-3 by E2F1 rather than through its traditional way. Transient transfection analysis and chromatin immunoprecipitation (ChIP) assays revealed that the overexpression of Rta elevated the expression of E2F1 and increased the binding of E2F1 to the promoter of IRF-3. The mutation of the E2F1-binding site and the knocking down of E2F1 by small interfering RNA (siRNA) can eradicate the inhibitory effect of Rta. These results suggested that Rta represses IRF-3 expression by increasing E2F1 binding to the IRF-3 promoter.
\end{abstract}

\section{Introduction}

To date, the human interferon regulatory factor (IRF) family has nine members (IRF1-9) (1). Of all the members of the IRF family, IRF-3 and IRF-7 are known to be the key regulators of the expression of Type I interferons (IFNs; IFN- $\alpha$ and IFN- $\beta$ ). IRF-3 is responsible for the early phase of Type I IFN induction. Together with IRF-7, IRF-3 is a critical element in the activation of host innate immune responses, particularly

Correspondence to: Professor Guo-Ping Zhou, Department of Pediatrics, The First Affiliated Hospital, Nanjing Medical University, 300 Guangzhou Road, Nanjing, Jiangsu 210029, P.R. China

E-mail: gpzhou2003@yeah.net

Key words: interferon regulatory factor 3, replication and transcription activator, E2F1 transcription factor in response to infection by different pathogens, including viruses $(2,3)$. In unstimulated cells, IRF-3 is constitutively present as a monomer in the cell cytoplasm. Viral infection can trigger the phosphorylation of IRF-3, mediated by the I $\mathrm{B}$ kinase (IKK)-related kinases, TANK-binding kinase 1 (TBK1) and IkappaB kinase epsilon (IKKe), resulting in its homodimerization and heterodimerization, nuclear localization and association with the coactivator CREB-binding protein (CBP)/p300. The IRF-3 complex, which is retained in the nucleus, induces transcription of IFN- $\beta$ and other genes (4-6).

Epstein-Barr virus (EBV) is a widespread human gamma-herpes virus. EBV is the causative agent of infectious mononucleosis and is closely associated with several human malignant diseases, including lymphoma, nasopharyngeal carcinoma, gastric carcinoma and lymphoproliferative diseases in immunocompromised patients (7). Se Thoe SY et al demonstrated that there was a positive association between EBV and carcinoma of the cervix (8). EBV expresses two transcription factors, replication and transcription activator (Rta) and BZLF1 transcription activator (Zta), during the immediate-early stage of the lytic cycle. The two proteins often collaborate to activate the transcription of EBV lytic genes synergistically. EBV Rta is known to mediate the switch from latent to lytic viral replication as well as other biological functions. It is able to activate a class of genes, cellular and viral. As is generally known, viruses commonly express abundant amounts of lytic proteins during the productive cycle. To achieve this, viruses often use viral-encoded transcription factors to promote the transcription of viral genes. Furthermore, these transcription factors may collaborate with each other to activate transcription synergistically and achieve high levels of expression of proteins that are required for viral lytic development. An earlier study demonstrated that EBV BRLF1 inhibited the transcription of IRF-3 and IRF-7 in HEK-293 cells and suppressed the induction of IFN- $\beta$ (9). The authors demonstrated that BRLF1 had the ability to evade host innate immune responses. However, the mechanisms underlying the regulation of IRF-3 by Rta remains unknown.

Previously $\mathrm{Xu}$ et al identified that the transcription factor E2F1 could repress the IRF-3 promoter activity by directly binding to its promoter $(10,11)$. Bioinformatics information 
demonstrates that there is no Rta-response element in the IRF-3 promoter. Thus, Rta may regulate IRF-3 by an indirect mechanism. In the present study, we demonstrated that exogenous Rta expression leads to a decrease in IRF-3 transcription and protein expression in HeLa cells. Rta also inhibits IRF-3 promoter activity. We demonstrated that E2F1 is crucial for the repression of the IRF-3 promoter by Rta through overexpression and mutation analysis, as well as the E2F1 small interfering RNA (siRNA) experiment. Rta was able to upregulate the expression of E2F1 and increase E2F1 interaction with the IRF-3 promoter. These results suggested that the repression of IRF-3 by the EBV immediate-early protein Rta may be mediated through E2F1 in HeLa cells.

\section{Materials and methods}

Cell culture. Human HeLa cells were maintained in Dulbecco's modified Eagle's medium containing 10\% heat-inactivated fetal bovine serum, supplemented with penicillin $(100 \mathrm{U} / \mathrm{ml})$ and streptomycin $(100 \mu \mathrm{g} / \mathrm{ml})$. Cells were incubated at $37^{\circ} \mathrm{C}$ with $100 \%$ humidity in $5 \% \mathrm{CO}_{2}$ and passaged using standard cell culture techniques. The study was approved by the ethics committee of The First Affiliated Hospital, Nanjing Medical University, Nanjing, China.

Plasmids and transfection. The cloning of the human IRF-3 gene promoter region was performed as described previously (10). The mutation of the putative E2F1 site at -109/-102 of the IRF-3 promoter was performed using the QuikChange Site-Directed Mutagenesis kit (Stratagene, La, Jolla, CA, USA). The sequence containing the E2F1 binding site was mutated from 5'-GTTCAACTTTCCCGCGCCTGC-3' to 5'-GTTCAACTTTAAAGCGCCTGC-3' (mutations shown in bold). The expression plasmids pcDNA-E2F1 (provided by Dr W. Douglas Cress) and the pcDNA empty vector were purified and were cotransfected using Lipofectamine ${ }^{\mathrm{TM}} 2000$ (Invitrogen Life Technologies, Carlsbad, CA, USA) and then incubated for $24 \mathrm{~h}$. The DNA fragment that encodes Rta was isolated from pET-Rta (provided by Dr Shih-Tung Liu) by NheI and HindIII digestion and inserted into the NheI and HindIII sites to yield plasmid pcDNA-Rta.

Double stranded siRNA. An RNA interference strategy was employed to silence endogenous E2F1 in HeLa cells. Double stranded siRNA specific for E2F1 and control siRNA were synthesized and were purified using high-performance liquid chromatography (Gene Pharma, Shanghai, China). The siRNA sequences used were as follows: (sense): 5'-GGCCCGAUCGAUGUUUUCC-3' for E2F1 (12) and 5'-CGUAAACGGCCACAAGUUC-3' for the control siRNA. siRNA oligonucleotides were transfected into cells at a concentration of $100 \mathrm{nM}$ using Lipofectamine ${ }^{\mathrm{TM}}$ (Invitrogen Life Technologies) according to the manufacturer's instructions.

Dual-luciferase reporter assays. Cells were seeded into 96-well plates $24 \mathrm{~h}$ prior to transfection. The Rta expression plasmid or the empty vector was individually cotransfected into HeLa cells, together with the appropriate IRF-3 promoter reporter plasmids or the E2F1 mutation plasmid using
Lipofectamine $^{\text {TM }} 2000$ (Invitrogen Life Technologies). The pRL-TK plasmid (Promega, Madison, WI, USA; 2 ng/sample) containing the Renilla luciferase gene driven by the herpes simplex virus thymidine kinase promoter was cotransfected with the constructs, and the luciferase activity was normalized. The preparation of cell lysates and measurements of luciferase activity were performed using the Dual-Luciferase Reporter Assay system (Promega) and TD-20/20 luminometer (Turner Designs, Sunnyvale, CA, USA) according to the manufacturer's instructions.

Chromatin immunoprecipitation assay (ChIP). The ChIP assay was performed using the ChIP-IT kit (Active Motif, Carlsbad, CA, USA) according to the manufacturer's instructions. Briefly, three $100 \mathrm{~cm}^{2}$ dishes of $80-90 \%$ confluent HeLa cells were treated with $1 \%$ formaldehyde in phosphate buffer solution (PBS) for $10 \mathrm{~min}$ at room temperature. The formaldehyde was inactivated by the addition of $0.125 \mathrm{M}$ of glycine in PBS to the cells for $5 \mathrm{~min}$ at room temperature. The cells were then washed in ice cold PBS and then lysed with lysis buffer containing $1 \%$ sodium dodecyl sulfate (SDS). Sonication of cross-linked chromatin was performed at $200 \mathrm{~W}$ with five rounds of $20 \mathrm{sec}$ pulses so that chromatin fragments which were obtained, ranged from 500 to $1,000 \mathrm{bp}$ in size. Soluble chromatin was subjected to overnight immunoprecipitation with anti-IgG or anti-E2F1 (C-20; Santa Cruz Biotechnology Inc., Santa Cruz, CA, USA). A portion of the chromatin solution was kept to check the amount of input DNA in different samples prior to immunoprecipitation. For each immunoprecipitation, $2 \mu \mathrm{g}$ of the appropriate antibody was incubated with a precleared chromatin aliquot overnight at $4^{\circ} \mathrm{C}$. Following immunoprecipitation and elution, the eluent was heated to $65^{\circ} \mathrm{C}$ for $6 \mathrm{~h}$ to reverse the cross-link, and then DNA was purified using minicolumns provided with the kit. The purified DNA was amplified by the promoter-specific primers ChIP-F, 5'-CACCCCTCGTCAACACCC-3' and ChIP-R, 5'-CGCGGGAAAGTTGAACTAATA-3' and quantitative reverse transcription polymerase chain reaction (RT-PCR) was performed according to the manufacturer's instructions.

RNA purification and quantitative real-time $R T-P C R$. Total RNA extraction was performed using TRIzol reagent followed by chloroform-isopropanol extraction and ethanol precipitation. Subsequently, duplicate samples of $1 \mu 1$ of each cDNA were used as a template. The quantification of gene transcripts was performed by real-time PCR using SYBR Green I dye (Invitrogen Life Technologies) and the ABI PRISM 7700 sequence detection system (PE Applied Biosystems, Wellesley, MA, USA). The specificity of amplification was assessed for each sample by melting curve analysis. Expression values were normalized with control GAPDH. The primers used were as follows: sense primer 5'-GTCGATCAAAAAGAAAGCCCCAGCG-3' and antisense primer 5'-CATCCTGCCGTAGGCCGTGCTTCC-3' for IRF-3; sense primer 5'-ATGTTTTCCTGTGCCCTGAG-3' and antisense primer 5'-ATCTGTGGTGAGGGATGAGG-3' for E2F1 and sense primer 5'-AGGTCGGAGTCAACGGAT-3' and antisense primer 5'-TCCTGGAAGATGGTGATG-3' for GAPDH. 
Western blot analysis. Samples were lysed in Laemmli buffer, boiled, electrophoresed on SDS-polyacrylamide gel and separated proteins were transferred onto polyvinylidene difluoride membranes. Membranes were incubated in 5\% dry milk in Tris-buffered saline with Tween-20 (TBST; $0.25 \mathrm{M}$ of Tris- $\mathrm{HCl} \mathrm{pH}$ 7.6, 0.19 $\mathrm{M}$ of $\mathrm{NaCl}$ and $0.1 \%$ Tween-20) for $1 \mathrm{~h}$ to block nonspecific sites. The primary antibodies used were mouse anti-GAPDH (Santa Cruz Biotechnology, Inc.), mouse anti-IRF-3 (3F10; Santa Cruz Biotechnology, Inc.) and rabbit anti-E2F1 (C-20; Santa Cruz Biotechnology, Inc.). Membranes were washed twice with TBST and treated with either a horseradish peroxidase-linked goat anti-mouse or anti-rabbit antibody. Reactive proteins were visualized by enhanced chemiluminescence (Pierce, Rockford, IL, USA).

Statistical analysis. The results were analyzed by using the paired two tailed student's-t test. $\mathrm{P}<0.05$ was considered to indicate a statistically significant difference.

\section{Results}

Rta suppresses IRF-3 mRNA and protein expression in HeLa cells. To investigate the effect of Rta on the regulation of IRF-3 expression, the Rta expression plasmid was adopted. HeLa cells were transfected with the Rta expression plasmid pcDNA-Rta or the corresponding control vector pcDNA. RNA and protein were extracted $24 \mathrm{~h}$ and $48 \mathrm{~h}$ after transfection, respectively. As shown in Fig.1, overexpression of Rta led to a $41 \%$ decrease of IRF-3 mRNA level and a $28 \%$ reduction of IRF-3 protein expression. These results suggested that EBV Rta could negatively regulate the expression of IRF-3 in HeLa cells.

Rta decreases the promoter activity of IRF-3 in HeLa cells. To explore whether Rta could regulate IRF-3 expression at the transcriptional level, we cloned a series of luciferase reporter plasmids containing different IRF-3 promoter truncations, spanning from -982 to +18 bp relative to the transcription start site, and transfected them into HeLa cells. As shown in Fig. 2, different promoter activities were observed in pGL3982, 624, 503, 285, 161 and 149, while pGL3-67 and pGL3-93 had little promoter activity. This result was similar to our previous study in HEK293 cells, which indicated that the region between -149 and -93bp was sufficient for full promoter activity. Notably, the promoter activities of all the plasmids which contain an E2F1-binding site were more or less reduced under exogenous Rta expression, which suggested that E2F1 may be important in the process of suppressing IRF-3 expression by Rta.

Repression of IRF-3 promoter activity by Rta is mediated by E2F1 in HeLa cells. To further confirm the mediation of E2F1 in the suppression of IRF-3 expression by Rta, we first performed an E2F1-site direct deletion mutation in pGL3-149. As shown in Fig. 3A, an E2F1-site direct deletion mutation completely eliminated the inhibitory action of Rta. Then, we overexpressed E2F1 by adopting an E2F1 expression plasmid, pcDNA-E2F1 and knocked down endogenous E2F1 using siRNA specific for E2F1. The overexpression and interference efficiency were detected by western blot analysis (data not shown). As depicted in Fig. 3B and Fig. 3C, cotransfection of
pGL3-149 with Rta/E2F1 resulted in a decline of 32\% compared with the Rta/pcDNA group. Cotransfection of pGL3-149 with Rta/E2F1 resulted in a decrease of $37 \%$ compared with the E2F1/pcDNA group. Cotransfection of pGL3-149 with pcDNA resulted in a 1.7-fold increase compared with the Rta/E2F1 group. However, cotransfection of pGL3-149 with Rta/E2F1 siRNA led to an increase of $50 \%$ compared with the Rta/control siRNA group. Cotransfection of pGL3-149 with pcDNA/E2F1 siRNA led to an increase of $114 \%$ compared with the Rta/E2F1 siRNA group. Cotransfection of pGL3-149 with pcDNA/control siRNA led to an increase of $76 \%$ compared with the Rta/control siRNA group. The results suggested that E2F1 was indispensable for the repression of the IRF-3 promoter activity by Rta.

Repression of the IRF-3 gene by Rta is mediated through E2FI in HeLa cells. To examine whether E2F1 could mediate the inhibitory action of Rta on IRF-3 mRNA levels, we transfected the Rta expression plasmid into HeLa cells with overexpression of E2F1. As shown in Fig. 4A, cotransfection of Rta with E2F1 led to a $31 \%$ decline compared with the pcDNA group, a $37 \%$ decline compared with the pcDNA/E2F1 group and a $56 \%$ decline compared with the pcDNA group. We also identified the expression of IRF-3 mRNA by Rta via knocking down E2F1 by siRNA. As displayed in Fig. 4B, cotransfection of Rta with E2F1 siRNA led to a $35 \%$ decline compared with the pcDNA/E2F1 siRNA group and a $25 \%$ decline compared with the Rta/control siRNA group. Cotransfection of Rta with control siRNA led to an increase of $40 \%$ compared with the pcDNA/control siRNA group. These results suggested that E2F1 is important in the repression of IRF-3 by Rta in HeLa cells.

Rta upregulates the expression of E2F1 in HeLa cells. The results demonstrated that Rta could repress IRF-3 expression through the transcription factor E2F1, however, whether Rta affects E2F1 expression is unclear. Thus, Rta was overexpressed in HeLa cells by the Rta expression plasmid. Notably, E2F1 mRNA and protein expression were markedly upregulated by the overexpression of Rta (Fig. 5), which indicated that Rta may repress IRF-3 expression through upregulating E2F1 levels.

E2F1 interaction with the IRF-3 promoter is increased by Rta. We identified that Rta could upregulate the expression of E2F1 in HeLa cells and inhibit IRF-3 expression. IRF-3 is negatively regulated by E2F1. To determine whether E2F1 interacting with the IRF-3 promoter was increased by Rta, a ChIP assay was performed. Fig. 6 demonstrated that anti-E2F1 antibodies precipitated proteins bound to the amplified sequence of the IRF-3 promoter by Rta was increased compared with the control, whereas nonspecific IgG (control antibody) failed to precipitate proteins bound to this sequence. Based on the experimental results, we concluded that Rta could suppress the expression of IRF-3 by increasing E2F1 binding to the IRF-3 promoter.

\section{Discussion}

The innate immune response is the first line of defense against invading viruses. IRF-3 has been implicated in virus and 


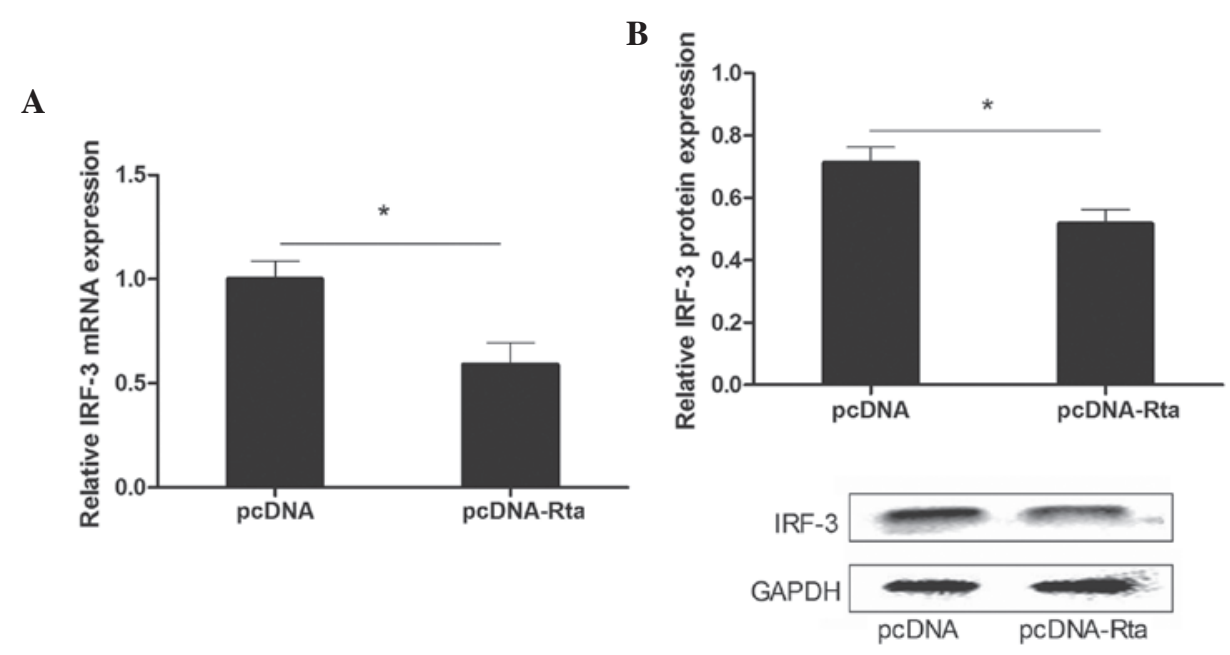

Figure 1. Rta suppresses IRF-3 gene expression in HeLa cells. (A) pcDNA-Rta and the vector control were transfected into HeLa cells, respectively. RNA was extracted $24 \mathrm{~h}$ later. Overexpression of Rta resulted in a decrease of IRF-3 by $41 \%$ compared with the vector control ("P $<0.05$ vs. pcDNA). (B) To investigate the effect of Rta on IRF-3 protein expression, protein was extracted $48 \mathrm{~h}$ after transfection. A lower level of IRF-3 was detected by western blot analysis. Overexpression of Rta resulted in a decrease of $28 \%$ compared with the vector control (" $\mathrm{P}<0.05 \mathrm{vs}$. pcDNA). The data from triplicate experiments and their averages are indicated in the figure. Error bars indicate standard deviation. Rta, replication and transcription activator; IRF-3, interferon regulatory factor 3.

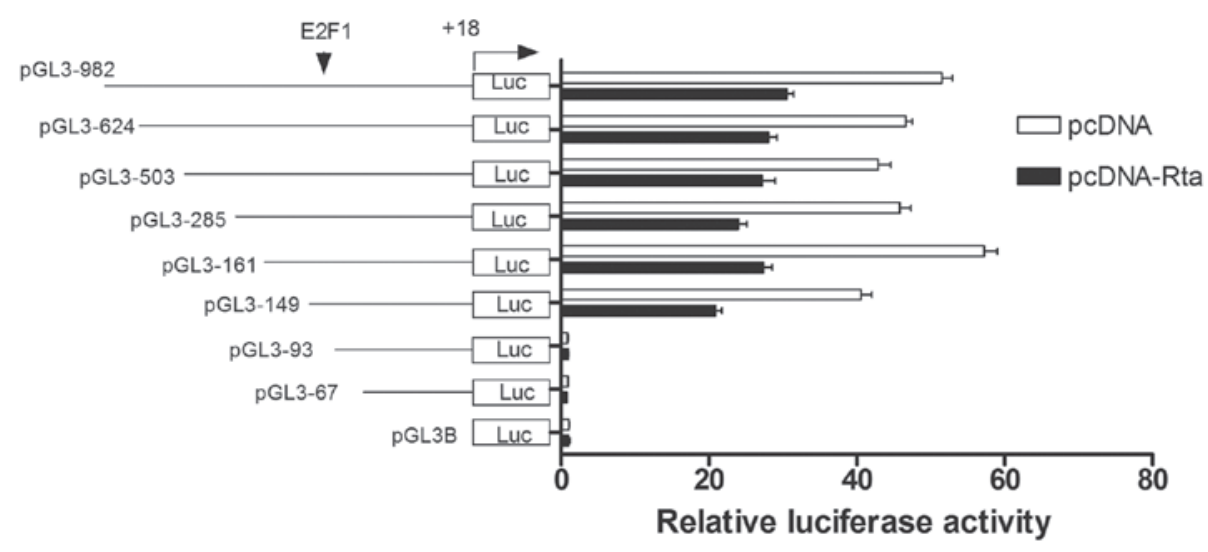

Figure 2. Effect of Rta on IRF-3 promoter activity in HeLa cells. HeLa cells were individually cotransfected with equal amounts (100 ng) of eight indicated IRF-3 promoter deletion constructs of Luc-reporter together with either the pcDNA empty vector (100 ng) or the Rta expressing vector pcDNA-Rta (100 ng). pRL-TK was also introduced into each transfected cell sample as an internal control. All IRF-3 promoter fragments except pGL3-93 and pGL3-67 which had no E2F1 binding site exhibited significant repression under exogenous Rta expression. The data were normalized for Renilla luciferase activity and represent the means and standard deviations of three independent experiments. The data of triplicate experiments and their averages are indicated in the figure. Error bars indicate standard deviation. Rta, replication and transcription activator; IRF-3, interferon regulatory factor 3; pRL-TK, renilla luciferase plasmid.

double-stranded RNA mediated induction of type I IFN and regulated on activation, normal $\mathrm{T}$ cell expressed and secreted (RANTES), in DNA damage signaling, in tumor suppression and in virus-induced apoptosis. EBV is a member of the human gamma-herpesvirus subfamily that infects over $90 \%$ of the global adult population. There is a wide variety of clinical syndromes with which persistent and/or chronic and/or latent EBV infection has been linked. Therefore, it is extremely difficult to identify an univocal pathogenetic link. In the present study, we demonstrated that Rta could repress the expression of IRF-3. Bentz GL et al (9) also identified that BRLF1 had the ability to suppress the expression of IRF-3 and evade host innate immune responses. This may be one of the EBV pathogenetic mechanisms.

The mechanisms of regulation of IRF-3 by other herpesviruses have been demonstrated. The immediate-early protein, ICP0 of bovine herpesvirus I and herpes simplex virus, inhibits the activity of IRF-3 by recruiting activated IRF-3 and inducing its degradation (13-15). The human cytomegalovirus encodes a protein, pp65, which subverts the activation of IRF-3 by inhibiting its nuclear accumulation and regulating innate immune responses (16). The IE protein 1 of the related $\beta$-herpesvirus HHV-6 also inhibits the nuclear localization of IRF-3 leading to decreased IFN- $\beta$ production (17). The majority of these findings explain the mechanisms at the protein level. There is little study concerning the regulation of IRF-3 at the transcriptional level. Promoters are key players in gene regulation. They receive signals from various sources and control the levels of transcription. Previously, we revealed that the transcription factor E2F1 could repress the expression of IRF-3. Further study demonstrated that it repressed IRF-3 by directly binding to its promoter (11). In the present study, we demonstrated that Rta could suppress IRF-3 expression through inhibiting the promoter activity of IRF-3 via the E2F1 
A
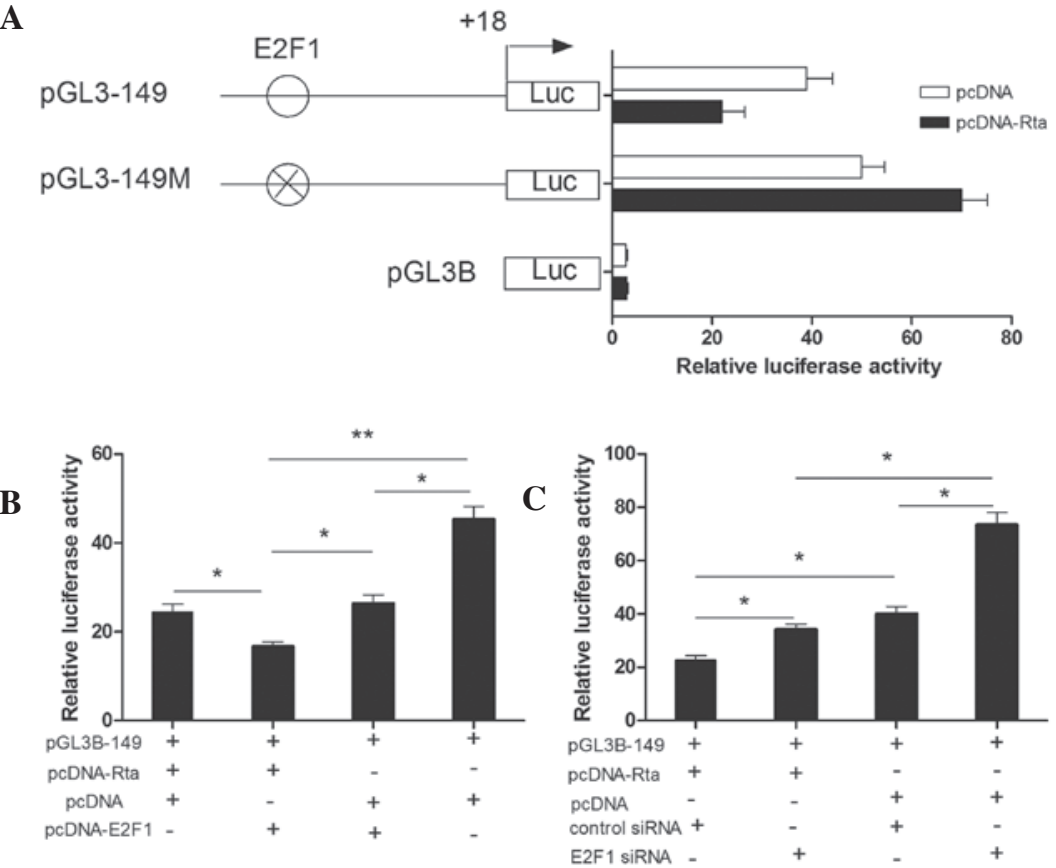

Figure 3. Repression of IRF-3 promoter activity by Rta is mediated through E2F1 in HeLa cells. (A) Rta and vector control were cotransfected with pGL3-149M and pGL3-149 into HeLa cells, respectively. Mutation of the E2F1 binding site was not able to decrease the promoter activity by Rta compared with the vector control. (B) Cotransfection of pGL3-149 with Rta/E2F1 resulted in a decrease of 32\% compared with the Rta/pcDNA group ("P<0.05 vs. Rta/pcDNA). Cotransfection of pGL3-149 with Rta/E2F1 resulted in a decrease of 37\% compared with the E2F1/pcDNA group ("P $<0.05$ vs. E2F1/pcDNA). Cotransfection of pGL3-149 with pcDNA resulted in a 1.7-fold increase compared with the Rta/E2F1 group ("*P<0.01 vs. Rta/E2F1). (C) Cotransfection of pGL3-149 with Rta/E2F1 siRNA led to an increase of 50\% compared with the Rta/control siRNA group ("P<0.05 vs. Rta/control siRNA). Cotransfection of pGL3-149 with pcDNA/E2F1 siRNA led to an increase of $114 \%$ compared with the group of Rta/E2F1 siRNA ("P<0.05 vs. Rta/E2F1 siRNA). Cotransfection of pGL3-149 with pcDNA/control siRNA led to an increase of $76 \%$ compared with the Rta/control siRNA group ("P $<0.05$ vs. Rta/control siRNA). The data of triplicate experiments and their averages were indicated in the figure. Error bars indicate SD (standard deviation). Rta, replication and transcription activator; IRF-3, interferon regulatory factor 3; siRNA, small interfering RNA.

A

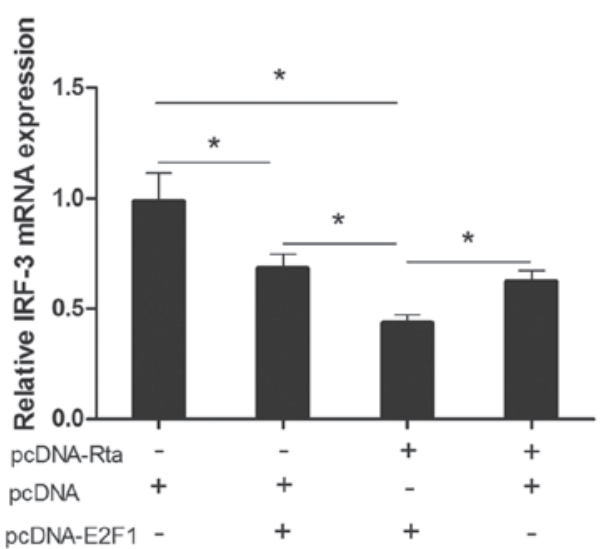

B

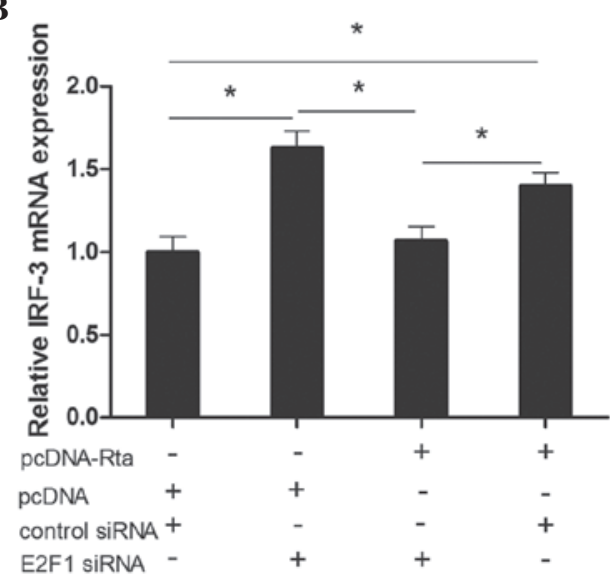

Figure 4. Repression of the IRF-3 gene by Rta is mediated through E2F1 in HeLa cells. (A) Cotransfection of Rta with E2F1 led to a 31\% decline compared with the pcDNA group ("P $<0.05$ vs. pcDNA), a 37\% decline compared with the pcDNA/E2F1 group ("P $<0.05$ vs. pcDNA/E2F1) and a $56 \%$ decline compared with the pcDNA/pcDNA group ("P<0.05 vs. pcDNA/pcDNA). (B) Cotransfection of Rta with E2F1 siRNA led to a 35\% decline compared with the pcDNA/E2F1 siRNA group ("P<0.05 vs. pcDNA/E2F1 siRNA), a $25 \%$ decline compared with the Rta/control siRNA group ("P $<0.05$ vs. Rta/control siRNA). Cotransfection of Rta with control siRNA led to an increase of $40 \%$ compared with the pcDNA/control siRNA group ("P $<0.05 \mathrm{vs.} \mathrm{pcDNA/control} \mathrm{siRNA).} \mathrm{The} \mathrm{data} \mathrm{from}$ triplicate experiments and their averages are indicated in the figure. Error bars indicate standard deviation. Rta, replication and transcription activator; IRF-3, interferon regulatory factor 3; siRNA, small interfering RNA.

site. We demonstrated the regulation of IRF-3 at the transcriptional level and this may be a new mechanism of regulation of IRF-3 by herpesviruses.

EBV Rta has two methods to regulate target genes. Firstly, it can activate genes by directly binding to a
GC-rich motif known as the Rta-responsive element (RRE), 5'-GNCCN ${ }_{9} \mathrm{GGNG}^{2} \mathrm{3}^{\prime}$ (18), found in viral promoters to activate the expression of BMRF1, BMLF1, and BALF2. It has been demonstrated that Rta binds directly to the early lytic EBV gene SM promoter and the interaction between Rta and CBP 
A

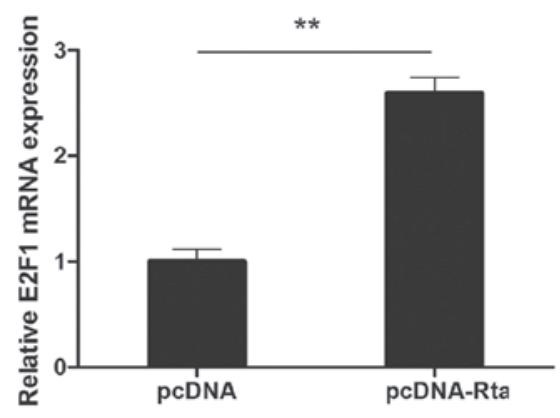

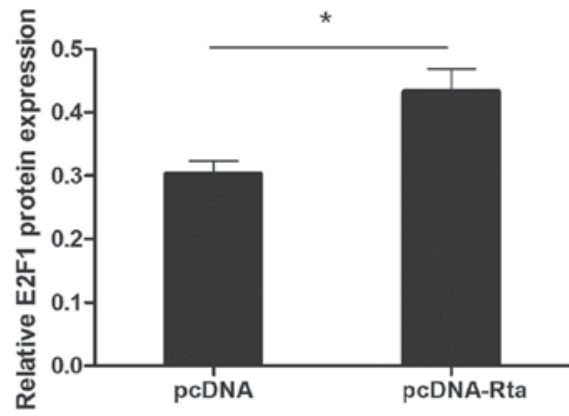

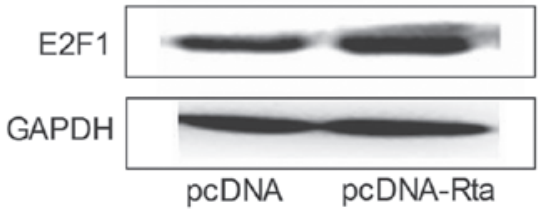

Figure 5. Rta upregulates the expression of E2F1 in HeLa cells. (A) Overexpression of Rta resulted in a marked increase in E2F1 mRNA by 1.6-fold compared with the vector control ( ${ }^{* *} \mathrm{P}<0.01$ vs. pcDNA). (B) Overexpression of Rta resulted in an increase of $40 \%$ compared with the vector control ("P<0.05 vs. pcDNA). The data from triplicate experiments and their averages are indicated in the figure. Error bars indicate standard deviation. Rta, replication and transcription activator.
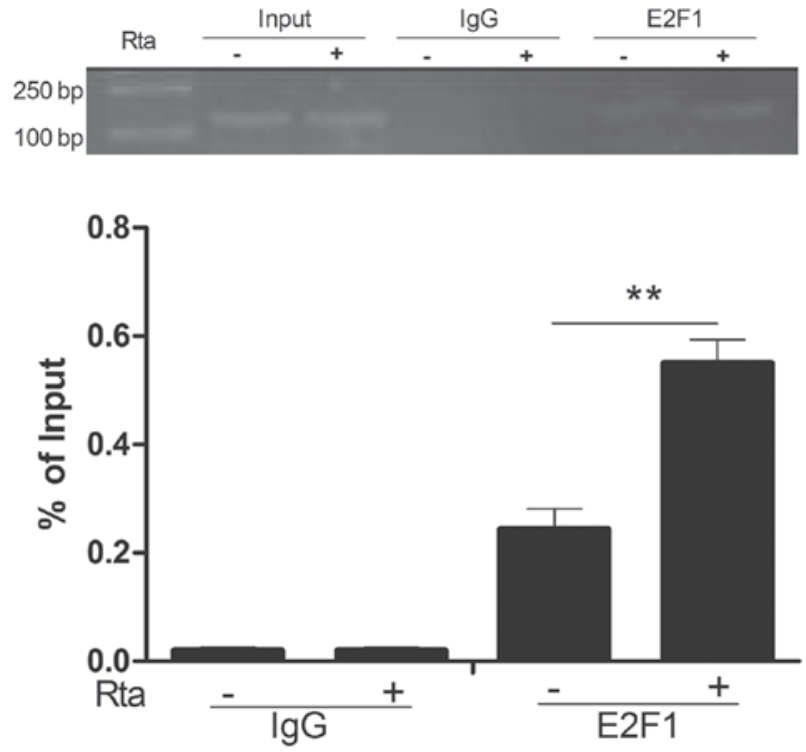

Figure 6. ChIP analysis was used to detect protein binding to the IRF-3 promoter in vivo, using antibodies to $\mathrm{E} 2 \mathrm{~F} 1$ and the $\mathrm{IgG}$ control. ChIP DNA was analyzed by real-time RT-PCR at the Rta or pcDNA open bars site in HeLa cells. Rta resulted in a marked increase of E2F1 binding to the IRF-3 promoter by 1.3 -fold compared with the vector control ( ${ }^{* *} \mathrm{P}<0.01 \mathrm{vs.}$. pcDNA) Error bars indicate standard deviation. ChIP, chromatin immunoprecipitation; Rta, replication and transcription activator; IRF-3, interferon regulatory factor 3; RT-PCR, reverse transcription polymerase chain reaction.

is important for Rta-induced activation of the SM gene in Raji cells (19). Rta could upregulate decoy receptor 3 expression by binding to its promoter (20). Rta could also activate a class of genes that lack any detectable RRE. For example, the EBV BRLF1 gene which encodes the Rta protein has no RRE and Rta could form a complex with Sp1 and MCAF1 on a Sp1-binding site to autoregulate the transcription of BRLF1 and to regulate several host genes in EBV-infected cells (21). Rta may activate a key early EBV promoter (pol) through USF and E2F (22). Bioinformatics information demonstrates that there is no RRE in IRF-3 promoter. We demonstrated that Rta repressed IRF-3 expression through the E2F1-site in the promoter of IRF-3, however not through its traditional way. Swenson et al (23) and Guo et al (24) demonstrated that Rta could increase E2F1 expression, which functions as a transcription factor that enhances cell proliferation by binding to the promoter region of several genes, including those that are involved in cell cycle regulatory activities and DNA replication. In the present study, we demonstrated that Rta could increase E2F1 expression and increase its interaction with the IRF-3 promoter to repress IRF-3 expression in HeLa cells. We may find a new mechanism of regulation of IRF-3 by Rta

It is known that EBV may be associated with cervical carcinoma. IRF-3 is important in tumor suppression. EBV Rta could repress IRF-3 by increasing E2F1 interaction with the IRF-3 promoter. These results suggested that $\mathrm{E} 2 \mathrm{~F} 1$ may mediate the onset of cervical carcinoma by EBV. The present study may contribute to revealing the mechanisms of regulation of IRF-3 by Rta and providing new treatments for EBV infection through designing drugs which can target the transcription factor E2F1.

\section{Acknowledgements}

This study was supported by The National Natural Science Foundation of China (no. 30872804 and no. 81170661 to GPZ), the Specialized Research Fund for the Doctoral Program of Higher Education (no. 20113234110010 to GPZ) and the Project Funded by the Priority Academic Program Development of Jiangsu Higher Education Institutions.

\section{References}

1. Eason DD, Shepherd AT and Blanck G: Interferon regulatory factor 1 tryptophan 11 to arginine point mutation abolishes DNA binding. Biochim Biophys Acta 1446: 140-144, 1999.

2. Honda K and Taniguchi T: IRFs: master regulators of signalling by Toll-like receptors and cytosolic pattern-recognition receptors. Nat Rev Immunol 6: 644-658, 2006. 
3. Honda K, Takaoka A and Taniguchi T: Type I interferon [corrected] gene induction by the interferon regulatory factor family of transcription factors. Immunity 25: 349-360, 2006

4. Weaver BK, Kumar KP and Reich NC: Interferon regulatory factor 3 and CREB-binding protein/p300 are subunits of doublestranded RNA-activated transcription factor DRAF1. Mol Cell Biol 18: 1359-1368, 1998.

5. Yoneyama M, Suhara W, Fukuhara Y, et al: Direct triggering of the type I interferon system by virus infection: activation of a transcription factor complex containing IRF-3 and CBP/p300 EMBO J 17: 1087-1095, 1998.

6. Lin R, Heylbroeck C, Pitha PM and Hiscott J: Virus-dependent phosphorylation of the IRF-3 transcription factor regulates nuclear translocation, transactivation potential, and proteasomemediated degradation. Mol Cell Biol 18: 2986-2996, 1998.

7. Young LS and Rickinson AB: Epstein-Barr virus: 40 years on. Nat Rev Cancer 4: 757-768, 2004.

8. Se Thoe SY, Wong KK, Pathmanathan R, Sam CK, Cheng HM and Prasad U: Elevated secretory IgA antibodies to Epstein-Barr virus (EBV) and presence of EBV DNA and EBV receptors in patients with cervical carcinoma. Gynecol Oncol 50: 168-172, 1993.

9. Bentz GL, Liu R, Hahn AM, Shackelford J and Pagano JS: Epstein-Barr virus BRLF1 inhibits transcription of IRF3 and IRF7 and suppresses induction of interferon-beta. Virology 402 $121-128,2010$.

10. Xu HG, Ren W, Lu C and Zhou GP: Characterization of the human IRF-3 promoter and its regulation by the transcription factor E2F1. Mol Biol Rep 37: 3073-3080, 2010.

11. Xu HG, Ren W, Zou L, Wang Y, Jin R and Zhou GP: Direct repression of the human IRF-3 promoter by E2F1. Immunogenetics 63: 189-196, 2011.

12. Rogoff HA, Pickering MT, Frame FM, et al: Apoptosis associated with deregulated E2F activity is dependent on E2F1 and Atm/Nbs1/Chk2. Mol Cell Biol 24: 2968-2977, 2004

13. Melroe GT, DeLuca NA and Knipe DM: Herpes simplex virus 1 has multiple mechanisms for blocking virus-induced interferon production. J Virol 78: 8411-8420, 2004.
14. Melroe GT, Silva L, Schaffer PA and Knipe DM: Recruitment of activated IRF-3 and CBP/p300 to herpes simplex virus ICP0 nuclear foci: Potential role in blocking IFN-beta induction. Virology 360: 305-321, 2007

15. Saira K, Zhou Y and Jones C: The infected cell protein 0 encoded by bovine herpesvirus 1 (bICP0) induces degradation of interferon response factor 3 and, consequently, inhibits beta interferon promoter activity. J Virol 81: 3077-3086, 2007.

16. Abate DA, Watanabe S and Mocarski ES: Major human cytomegalovirus structural protein pp 65 (ppUL83) prevents interferon response factor 3 activation in the interferon response. J Virol 78: 10995-11006, 2004

17. Jaworska J, Gravel A, Fink K, Grandvaux N and Flamand L: Inhibition of transcription of the beta interferon gene by the human herpesvirus 6 immediate-early 1 protein. J Virol 81: 5737-5748, 2007.

18. Gruffat $\mathrm{H}$ and Sergeant A: Characterization of the DNA-binding site repertoire for the Epstein-Barr virus transcription factor R. Nucleic Acids Res 22: 1172-1178, 1994.

19. Swenson JJ, Holley-Guthrie E and Kenney SC: Epstein-Barr virus immediate-early protein BRLF1 interacts with CBP, promoting enhanced BRLF1 transactivation. J Virol 75: 6228-6234, 2001.

20. Ho CH, Hsu CF, Fong PF, et al: Epstein-Barr virus transcription activator RTA upregulates decoy receptor 3 expression by binding to its promoter. J Virol 81: 4837-4847, 2007.

21. Chang LK, Chung JY, Hong YR, et al: Activation of Sp1-mediated transcription by RTA of Epstein-Barr virus via an interaction with MCAF1. Nucleic Acids Res 33: 6528-6539, 2005.

22. Liu C, Sista ND and Pagano JS: Activation of the Epstein-Barr virus DNA polymerase promoter by the BRLF1 immediate-early protein is mediated through USF and E2F. J Virol 70: 2545-2555, 1996.

23. Swenson JJ, Mauser AE, Kaufmann WK and Kenney SC: The Epstein-Barr virus protein BRLF1 activates S phase entry through E2F1 induction. J Virol 73: 6540-6550, 1999.

24. Guo Q, Sun X, Yuan C, Zhou H, Li Y, Jie G and Jiang G: Effect of Rta protein of Epstein-Barr virus on the cell cycle in HeLa cells. Acta Virol 55: 311-316, 2011. 